Reprod. Nutr. Dévelop., 1983, 23 (4), 727-739.

\title{
Estimation in vitro à l'aide du phosphore radioactif des besoins en phosphore des microorganismes du rumen
}

\author{
Michelle DURAND, P. BEAUMATIN, Christiane DUMAY
}

Station de Recherches de Nutrition, I.N.R.A., 78350 Jouy-en-Josas, France.

Summary. In vitro estimate of rumen microbial requirements for phosphorus using ${ }^{32}$ P-labelled phosphate.

Microbial requirements for $P$ were assumed to be a function of the amount of microbial protein synthesis (microbial growth) and of the quantity of organic matter (OM) fermented in the rumen. The relationships among $P$ incorporation into microbial matter and protein synthesis, ammonia utilization, volatile fatty acid (VFA) production and organic matter fermented (OMF) were studied in short-term incubations $(3 \mathrm{~h})$ using ${ }^{32} \mathrm{P}$-labelled phosphate. The amount of $\mathrm{P}$ incorporated was calculated from extracellular phosphate pool specific activity and the radioactivity incorporated into the microbial sediment during incubation (table 1). The inocula came from sheep fed a protein-free purified diet. In order to vary the intensity of fermentation, carbohydrates with a wide range of degrees of enzymatic susceptibility were used as substrates and the medium was either provided or was deficient in $S$ and trace elements (table 4). Nitrogen was supplied as ammonium salts.

Linear regression analyses showed that $\mathrm{P}$ incorporation was positively correlated with the criteria of protein synthesis and $O M$ fermentation (figs. 1, 2, 3, 4). However, there was significant phosphorus incorporation when the value for nitrogen incorporation was zero (equation $\mathrm{A}$ : ( $\mathrm{Pi}(\mathrm{mg})=0.162 \mathrm{NH}_{3}-\mathrm{N}+0.376 ; \mathrm{r}=0.9$ ). This was assumed to result either from energetic uncoupling (fermentation without concomitant bacterial growth) or from the lysis of cold microbial cells only. Equation $A$ would reflect total $P$ incorporation and equation $\mathrm{A}^{\prime} \mathrm{Pi}(\mathrm{mg})=0.162 \mathrm{NH}_{3}-\mathrm{N}(\mathrm{mg})$, net $\mathrm{P}$ incorporation.

It was assumed that in vitro microbial requirements for $P$ were in the range of 30 $70 \mathrm{mg}$ of $\mathrm{P} /$ /iter of medium for 3-hour incubation, depending on the intensity of fermentation. Form a mean value of microbial $\mathrm{N}$ yield of $30 \mathrm{~g} / \mathrm{kg}$ of DOMR (organic matter apparently digested in the rumen), it was calculated that the total and net $\mathrm{P}$ requirements in vivo were 6 and $4.9 \mathrm{~g} / \mathrm{kg}$ of DOMR, respectively, corresponding to 3.9 and $3.2 \mathrm{~g} / \mathrm{kg}$ of DOM (digestible organic matter). From equation $D$, relating $P i$ to $O M F$, the $P$ requirements were about $4.4 \mathrm{~g} / \mathrm{kg}$ of DOM. It is suggested that microbial requirements for $P$ varied from 3 to $5 \mathrm{~g}$ of $\mathrm{P} / \mathrm{kg}$ of DOM, depending on the efficiency of microbial synthesis and the extent of carbohydrate fermentation. These results, considered as indicative, should be checked in in vivo experiments. 


\section{Introduction.}

Le phosphore $(\mathrm{P})$ est un élément essentiel pour tous les microorganismes. II intervient dans de nombreux systèmes enzymatiques particulièrement importants pour la fermentation des glucides et entre dans la composition du matériel cellulaire (acides nucléiques du ribosome, phospholipides membranaires, acide téichoïque des parois des bactéries Gram + ...). Des polyphosphates peuvent également être stockés dans la cellule quand l'énergie disponible est supérieure aux besoins de croissance ou quand la synthèse cellulaire est réduite en raison de l'absence d'un nutriment essentiel (Saizeva, Belozersky et Novojilova, 1959 ; Levy, Campbell et Blackburn, 1973).

La teneur en $P$ total des microorganismes du rumen est assez variable selon les auteurs. Hungate (1966) estime qu'elle est comprise entre 2 et $6 \%$ de la matière sèche (MS) et Stewart (1975), pour des bactéries en culture pure, entre 2 et $2,8 \%$. Sur des prélèvements effectués in vivo, les valeurs se situent en général entre 1 et $2 \%$ (Durand, non publié ; Martinez, 1972 ; Czerkawski, 1976 ; Van Nevel et Demeyer, 1977). Les valeurs observées du rapport N/P bactérien sont variables. Van Nevel et Demeyer (1977) notent une valeur moyenne de 8,37 $\pm 0,75$ dans le cas de contenu de rumen prélevé sur des moutons à jeun et, pour des échantillons prélevés à différents temps au cours de la journée, Czerkawski (1976) observe une valeur de 7,14 pour les protozoaires et les grandes bactéries et seulement de 3,85 pour les petites bactéries. En tenant compte des proportions relatives de ces microorganismes dans le contenu, le rapport pour la biomasse totale serait de 5,3 .

La distribution du $P$ cellulaire évolue au cours des incubations in vitro (Van Nevel et Demeyer, 1977). De même, in vivo, Palfii (1976) remarque au cours de la journée, chez le taurillon, des variations de la répartition des divers composés phosphorés du culot bactérien ( $P$ inorganique, $P$ lipidique, $P$ ribonucléique). L'apport de substrats au niveau du rumen n'étant pas constant, le taux de croissance bactérien peut varier. Or, Tempest (1969) a montré qu'une élévation du taux de croissance bactérien s'accompagnait d'un accroissement des teneurs en ARN et $P$ total des bactéries. Aussi, la détermination de la teneur en $P$ des microorganismes du rumen ne permet pas d'estimer les besoins moyens en cet élément.

Nous disposons actuellement de peu de données concernant l'estimation de ces besoins, notamment in vivo. In vitro, plusieurs auteurs ont tenté d'évaluer les quantités de $P$ disponible dans le milieu, permettant d'optimaliser la croissance des bactéries du rumen en culture pure ou l'activité cellulolytique du contenu (Durand et Kawashima, 1980). Les valeurs observées sont assez variables et se situent le plus souvent entre 20 et $100 \mathrm{mg} \mathrm{P} /$ litre de milieu. Cependant, des valeurs beaucoup plus élevées ont été suggérées telles que : 300 à $600 \mathrm{mg} P / /$ (Bonilla, 1976) pour la croissance et 1,5 g P/I (Demeyer, 1981) pour l'activité des cellulases.

L'objectif de la présente expérience a été de relier l'incorporation de phosphore à la synthèse de protéine microbienne, à l'utilisation de l'azote ammoniacal et à la production d'acides gras volatils, en utilisant du ${ }^{32} \mathrm{P}$ au cours d'incubations dont l'activité fermentaire varie selon la nature du substrat glucidique. 


\section{Matériel et méthodes.}

\section{Incubation.}

La méthode générale d'incubation et de traitement des échantillons est indiquée dans le tableau 1.

Le contenu de rumen servant d'inoculum pour les incubations a été prélevé sur quatre moutons mâles, castrés, de race lle-de-France, pesant en moyenne $60 \mathrm{~kg}$ et portant une fistule permanente du rumen. Les animaux recevaient $800 \mathrm{~g}$ par jour d'un aliment purifié sans protéine (tabl. 2) et $80 \mathrm{~g}$ de paille, distribués en deux repas. Les contenus de rumen, prélevés avant le repas du matin, ont été mélangés, filtrés sur gaze et dilués (sauf dans un cas) à parties égales avec une solution tampon dont la composition est indiquée dans le tableau 3 . Le volume final du milieu d'incubation était voisin de $50 \mathrm{ml}$.

Afin de faire varier l'activité fermentaire, plusieurs types de substrats glucidiques, différant par leur accessibilité aux enzymes, ont été utilisés et les milieux ont été ou non supplémentés en soufre et en oligo-éléments (tabl. 4) ; dans les milieux carencés le soufre et les oligo-éléments étaient absents du mélange tam-

\section{TABLEAU 1}

Mesure de I'incorporation de phosphore par la méthode ${ }^{32} P$

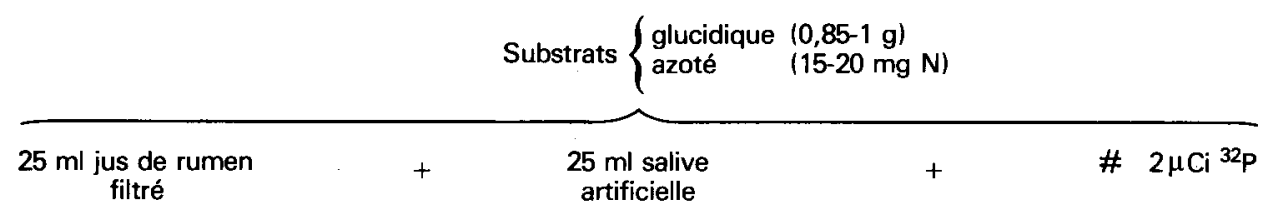

Aux temps 0 et $3 \mathrm{~h}$

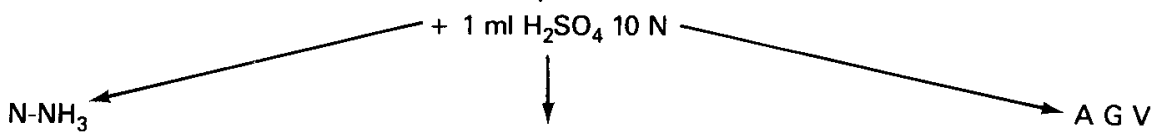

Centrifugation

$20000 \mathrm{~g}-20 \min$ à $4{ }^{\circ} \mathrm{C}$

+5 lavages culot

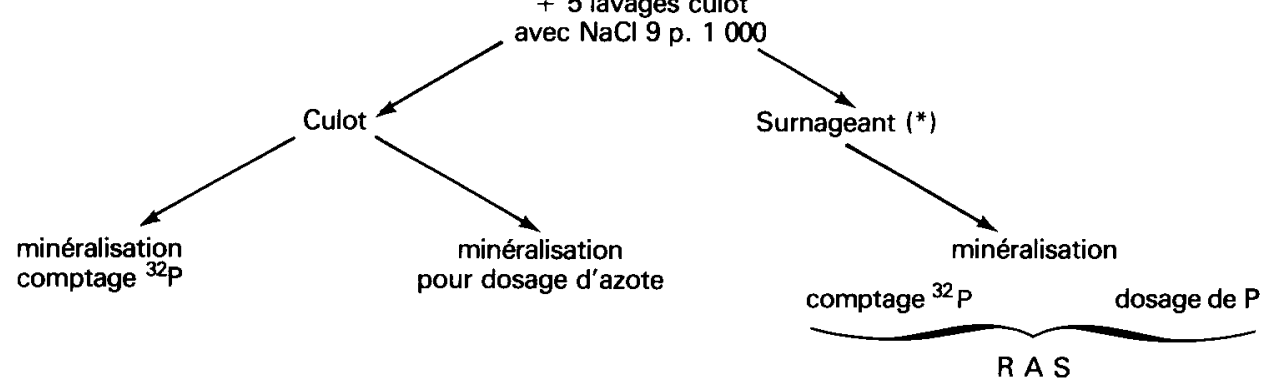

(*) Effectué au temps 0 uniquement. 
TABLEAU 2

Composition de l'aliment purifié de base

en $\%$

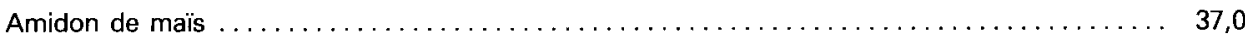

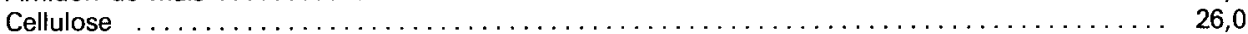

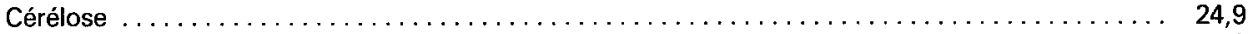

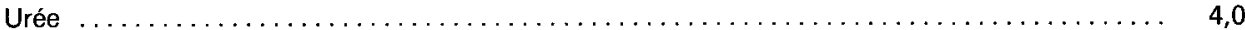

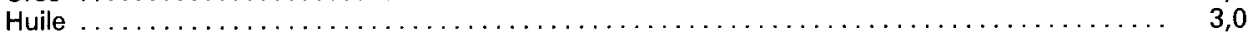

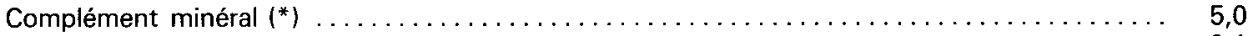

Chlorure de choline $\ldots \ldots \ldots \ldots \ldots \ldots \ldots \ldots \ldots \ldots \ldots \ldots \ldots \ldots \ldots \ldots \ldots \ldots, 0,1$

(*) Composition du complément minéral (en \%) : magnésie : 6,00; carbonate de calcium : 19,30; chlorure de potassium : 9,10 ; sulfate de sodium: 12,10 ; phosphate bicalcique : 22,50 ; phosphate monopotassique : 13,76 ; sulfate de potassium : 4,60 ; sulfate de magnésium, $7 \mathrm{H}_{2} \mathrm{O}$ : 9,10; chlorure de sodium : 0,70 ; sulfate de zinc, $7 \mathrm{H}_{2} \mathrm{O}: 0,50$; sulfate de cuivre, $5 \mathrm{H}_{2} \mathrm{O}: 0,091$; sulfate de cobalt, $7 \mathrm{H}_{2} \mathrm{O}: 0,006$; sulfate de manganèse, $\mathrm{H}_{2} \mathrm{O}: 0,760$; sulfate de fer, $7 \mathrm{H}_{2} \mathrm{O}: 1,4807$; sélénite de sodium, $5 \mathrm{H}_{2} \mathrm{O}: 0,0008$; molybdate d'ammonium, $4 \mathrm{H}_{2} \mathrm{O}: 0,0005$; iodure de potassium : 0,0010 .

\section{TABLEAU 3}

Composition du mélange tampon d'incubation

$\begin{array}{lllc} & \mathrm{g} / 1 & & \mathrm{mg} / \mathrm{l} \\ \mathrm{NaHCO}_{3} & 9,240 & \mathrm{FeSO}_{4}, 7 \mathrm{H}_{2} \mathrm{O} & 36,80 \\ \mathrm{Na}_{2} \mathrm{HPO}_{4}, 12 \mathrm{H}_{2} \mathrm{O} & 7,125 & \mathrm{MnSO}_{4}, \mathrm{H}_{2} \mathrm{O} & 18,90 \\ \mathrm{NaCl} & 0,470 & \mathrm{ZnSO}_{4}, 7 \mathrm{H}_{2} \mathrm{O} & 13,20 \\ \mathrm{KCl} & 0,450 & \mathrm{CuSO}_{4}, 5 \mathrm{H}_{2} \mathrm{O} & 2,16 \\ \mathrm{Na}_{2} \mathrm{SO}_{4} & 0,400 & \mathrm{CoCl}_{2}, 6 \mathrm{H}_{2} \mathrm{O} & 0,12 \\ \mathrm{CaCl}_{2} \text { anhydre } & 0,055 & \mathrm{Mo}\left(\mathrm{NH}_{4}\right)_{6} \mathrm{O}_{24}, 4 \mathrm{H}_{2} \mathrm{O} & 0,087 \\ \mathrm{MgCl}_{2} \text { anhydre } & 0,047 & \mathrm{Nal} & 0,016\end{array}$

pon. L'azote était apporté sous forme de carbonate ou de sulfate d'ammonium. Les incubations ont été réalisées dans des flacons à plasma de $125 \mathrm{ml}$ préalablement saturés de $\mathrm{CO}_{2}$ dans lesquels environ $2 \mu \mathrm{Ci}$ de ${ }^{32} \mathrm{P}$, apportés sous forme de $\mathrm{Na}_{2} \mathrm{HPO}_{4}$, ont été ajoutés au début de l'incubation. Les flacons ont été placés dans un bain-marie à agitation à $39^{\circ} \mathrm{C}$ et maintenus pendant des temps variant de 1 à $5 \mathrm{~h}$ (tabl. 4). Les fermentations ont été arrêtées par addition dans le milieu de $1 \mathrm{ml}$ de $\mathrm{H}_{2} \mathrm{SO}_{4} 10 \mathrm{~N}$. Des milieux témoins non incubés ont subi le même traitement et constituaient le temps 0 .

\section{Incorporation de $P$ dans le culot bactérien.}

La méthode utilisée est adaptée de celle décrite par Van Nevel et Demeyer (1977).

Les culots bactériens des temps 0 et de fin d'incubation ont été remis en suspension dans de l'eau distillée, homogénéisés avec un appareil Ultra-Turrax. Une partie aliquote a été minéralisée par voie sèche. Les cendres ont été reprises successivement avec $\mathrm{HCl}$ pur et $\mathrm{HNO}_{3}$ à $10 \%$ selon la méthode classique de dosage du phosphore. Une partie aliquote des surnageants de centrifugation a 
été minéralisée et traitée selon la méthode précédente. Les comptages de radioactivité ont été effectués sur $10 \mathrm{ml}$ de minéralisat en utilisant l'effet Cerenkov, à l'aide d'un compteur à scintillation liquide Nuclear Chicago. Les mesures de radioactivité ont porté sur la source radioactive, les surnageants au temps 0 (calcul de la radioactivité spécifique), les culots bactériens au début et à la fin de l'incubation.

La quantité de $\mathrm{P}$ incorporé dans les culots bactériens a été calculée selon la formule suivante :

$P$ incorporé $(\mathrm{mg})=\frac{\text { radioactivité dans le culot au temps } t}{\text { radioactivité spécifique du pool soluble de } P}$

La radioactivité mesurée dans le culot au temps $t$ a été corrigée par la radioactivité fixée par adsorption non enzymatique sur le culot au temps 0 , cette dernière représentant en moyenne $6,5 \%$ de la radioactivité incorporée dans les flacons incubés.

\section{Critères de l'activité microbienne.}

Les critères retenus ont été les suivants :

- azote protéique synthétisé (différence entre les valeurs au temps $t$ et au temps 0) ;

- utilisation de l'azote ammoniacal $\left(\mathrm{N}-\mathrm{NH}_{3}\right)$ [différence entre les valeurs au temps 0 (substrat + inoculum) et au temps $t$ ] ;

- production d'acides gras volatils (AGV) (différence entre les valeurs au temps $t$ et au temps 0 ).

La matière organique fermentée (MOF) est déduite des acides gras volatils formés dans le milieu d'incubation selon la formule suivante proposée par Van Nevel et Demeyer (1977) :

mmoles d'hexose $=\frac{\text { acétate }}{2}+\frac{\text { propionate }}{2}+$ butyrate $+\frac{\text { lactate }}{2}$

Au temps $t$, nos milieux ne contenant pas d'acide lactique, nous n'avons tenu compte que des AGV. Un poids moléculaire moyen de 162 a été utilisé pour calculer le poids d'hexose fermenté.

Le rendement de la protéosynthèse microbienne a été estimé en rapportant $\mathrm{N}-\mathrm{NH}_{3}$ utilisé ou $\mathrm{N}$-protéique synthétisé à la matière organique fermentée (MOF).

\section{Méthodes analytiques.}

Le phosphore a été dosé par colorimétrie au phosphovanadomolybdate d'ammonium (réaction de Misson). L'azote protéique, après précipitation par $\mathrm{H}_{2} \mathrm{SO}_{4}(10 \mathrm{~N})$ a été dosé par la méthode Kjeldahl. L'azote ammoniacal a été dosé à l'autoanalyseur (Technicon) après déprotéinisation des échantillons par l'acide trichloracétique à 2,5\% (Beaumatin, 1981). Les acides gras volatils ont été déterminés par chromatographie en phase gazeuse sur colonne de verre avec du chromosorb W/AW comme phase stationnaire et du SP1200 $10 \%+\mathrm{H}_{3} \mathrm{PO}_{4}$ $1 \%$ comme phase liquide (Ottenstein et Bartley, 1971). 


\section{Résultats.}

Les moyennes ( \pm écart type) des résultats de chaque essai sont rapportées au tableau 4.

Pour chacun de ces essais, nous avons obtenu 4 à 6 résultats correspondant à des incubations réalisées à partir d'un même inoculum.

Pour une incorporation faible de $P$ (cas de substrats peu fermentescibles), le coefficient de variation peut atteindre $18 \%$. En revanche, pour des incorporations supérieures à $1 \mathrm{mg}$ par fiole d'incubation $(50 \mathrm{ml})$, le coefficient de variation se situe généralement entre 4,6 et $5,3 \%$. Dans ces conditions, la méthode paraît fiable.

\section{TABLEAU 4}

Estimation in vitro à l'aide de ${ }^{32} P$ des besoins en $P$ des microorganismes du rumen

Moyenne des résultats par flacon $(50 \mathrm{ml}$ de milieu)

obtenus à partir d'un même inoculum pour un même traitement

\begin{tabular}{|c|c|c|c|c|c|}
\hline $\begin{array}{c}\text { Nature } \\
\text { du substrat } \\
\text { énergétique }\end{array}$ & $\begin{array}{c}\text { Conditions } \\
\text { d'incubation }\end{array}$ & $\begin{array}{l}P \text { incorporé } \\
\text { (mg) } \\
\pm \text { écart type }\end{array}$ & $\begin{array}{c}\mathrm{N}-\mathrm{NH}_{3} \\
\text { utilisé } \\
\text { (mg) }\end{array}$ & $\begin{array}{l}\text { N protéique } \\
\text { synthétisé } \\
\text { (mg) }\end{array}$ & $\begin{array}{c}\text { AGV } \\
\text { produits } \\
\text { (mmoles) }\end{array}$ \\
\hline $\begin{array}{c}\text { Sans substrat } \\
\text { AT } \\
\text { AT } \\
\text { AT } \\
\text { AT } \\
\text { AT } \\
\text { AT } \\
\text { AT } \\
\text { AT } \\
\text { AT } \\
\text { AT } \\
\text { ATex } \\
\text { ATex } \\
\text { AM } \\
\text { AM } \\
\text { AMex } \\
\text { AMex } \\
\text { ALp } \\
\text { ALp } \\
\text { ALp } \\
\text { ALp } \\
\text { M } \\
\text { M } \\
\text { M } \\
\text { M }\end{array}$ & $\begin{array}{l}1, \mathrm{c} \\
1, \mathrm{a} \\
1, \mathrm{~b} \\
1, \mathrm{c} \\
1, \mathrm{c} \\
3, \mathrm{c} \\
2, \mathrm{c} \\
4, \mathrm{c} \\
4, \mathrm{c} \\
1, \mathrm{c} \\
1, \mathrm{c} \\
1, \mathrm{c} \\
1, \mathrm{c} \\
1, \mathrm{c} \\
1, \mathrm{c} \\
1, \mathrm{c} \\
1, \mathrm{c} \\
2, \mathrm{c} \\
3, \mathrm{c} \\
3, \mathrm{c} \\
3, \mathrm{c} \\
1, \mathrm{c} \\
1, \mathrm{c} \\
1, \mathrm{~d} \\
1, \mathrm{~d}\end{array}$ & $\begin{array}{l}0,06 \pm 0,03 \\
0,10 \pm 0,02 \\
0,28 \pm 0,02 \\
0,48 \pm 0,04 \\
1,92 \pm 0,12 \\
1,68 \pm 0,07 \\
1,27 \pm 0,06 \\
1,32 \pm 0,07 \\
1,01 \pm 0,04 \\
2,46 \pm 0,06 \\
1,43 \pm 0,07 \\
2,16 \pm 0,10 \\
2,51 \pm 0,12 \\
1,03 \pm 0,05 \\
0,64 \pm 0,14 \\
1,23 \pm 0,05 \\
1,39 \pm 0,02 \\
1,11 \pm 0,05 \\
1,64 \pm 0,08 \\
1,59 \pm 0,06 \\
1,45 \pm 0,11 \\
1,40 \pm 0,05 \\
1,97 \pm 0,09 \\
2,53 \pm 0,11 \\
2,26 \pm 0,09\end{array}$ & $\begin{array}{r}1,69 \\
1,11 \\
1,85 \\
2,40 \\
9,80 \\
8,33 \\
5,00 \\
4,80 \\
4,07 \\
9,86 \\
5,45 \\
12,25 \\
10,53 \\
6,05 \\
2,03 \\
9,20 \\
6,93 \\
2,74 \\
4,75 \\
4,27 \\
4,58 \\
6,30 \\
12,10 \\
13,80 \\
10,90\end{array}$ & $\begin{array}{r}1,83 \\
1,49 \\
-\quad 0,20 \\
2,42 \\
10,06 \\
9,12 \\
5,70 \\
7,31 \\
4,68 \\
11,45 \\
7,11 \\
13,38 \\
11,57 \\
3,65 \\
2,59 \\
5,22 \\
8,86 \\
4,99 \\
8,73 \\
7,03 \\
7,79 \\
5,20 \\
8,38 \\
8,30 \\
4,17\end{array}$ & $\begin{array}{l}0,00 \\
0,58 \\
0,85 \\
1,11 \\
2,99 \\
3,28 \\
2,77 \\
2,86 \\
2,36 \\
3,25 \\
1,64 \\
4,76 \\
3,87 \\
1,39 \\
1,31 \\
2,12 \\
2,30 \\
2,50 \\
2,59 \\
2,71 \\
2,78 \\
2,39 \\
2,42 \\
3,33 \\
3,58\end{array}$ \\
\hline
\end{tabular}

$1=$ salive artificielle + oligo-éléments ; 2 = salive artificielle sans oligo-élément ni soufre ; $3=$ salive artificielle sans oligo-élément + soufre; $4=$ sans salive artificielle.

$\mathrm{AT}=$ Amidon de maïs témoin $;$ Atex $=$ Amidon de maïs témoin extrudé $; A M=$ Amidon de maïs riche en amylose ; AMex $=$ Amidon de maïs riche en amylose extrudé ; ALp $=$ Aliment purifié ; $M=$ Manioc.

Durée de l'incubation : $a=1 \mathrm{~h} ; \mathrm{b}=2 \mathrm{~h} ; \mathrm{c}=3 \mathrm{~h} ; \mathrm{d}=5 \mathrm{~h}$. 


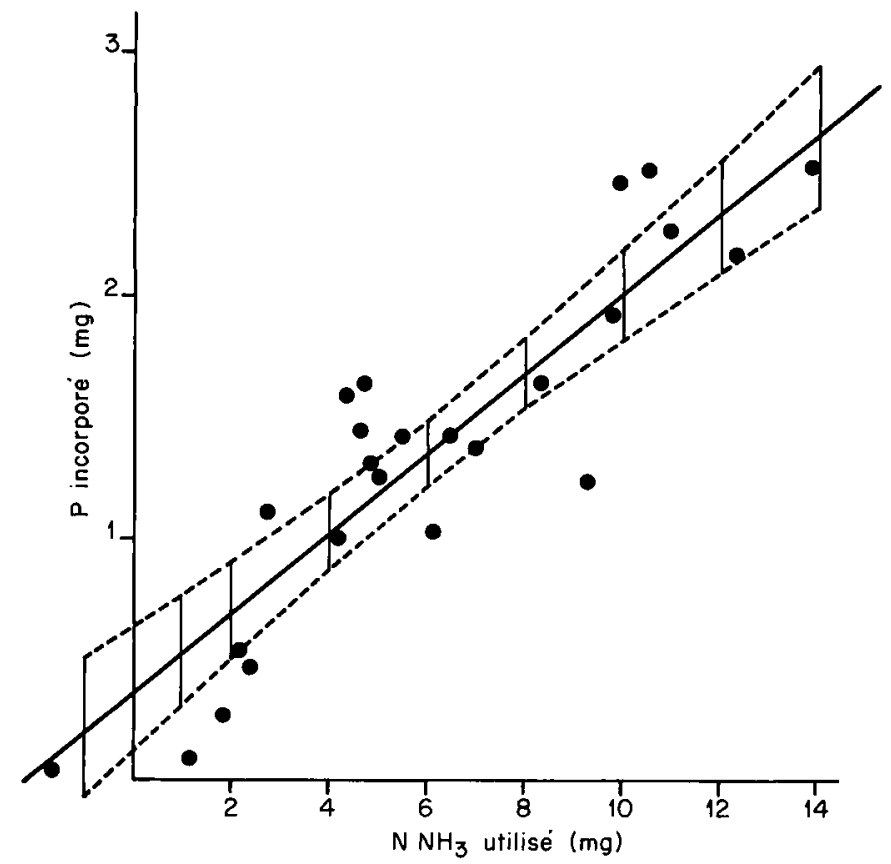

FIG. 1. - Relation entre l'incorporation de phosphore dans les bactéries (calculée à partir du ${ }^{32} \mathrm{P}$ ) et l'utilisation du $\mathrm{N}-\mathrm{NH}_{3}$

$\mathrm{Pi}_{(\mathrm{mg})}=0,162 \mathrm{~N}-\mathrm{NH}_{3(\mathrm{mg})}+0,376$.

$n=25 ; r=0,90 ;$ Syx $=0,321$.

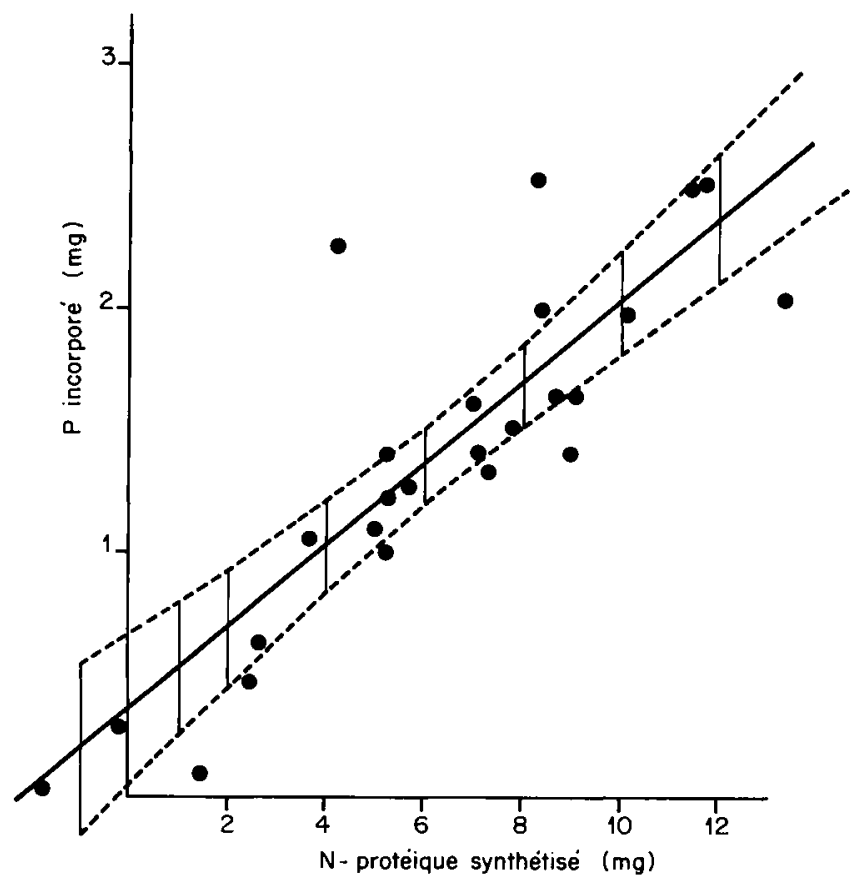

FIG. 2. - Relation entre l'incorporation de phosphore dans les bactéries (calculée à partir du ${ }^{32} \mathrm{P}$ ) et l'accroissement de l'azote protéique bactérien.

$\mathrm{Pi}_{(\mathrm{mg})}=0,166 \mathrm{~N}$-prot $\mathrm{t}_{(\mathrm{mg})}+0,355$.

$\mathrm{n} \stackrel{\mathrm{m}}{=} 25 ; \mathrm{r}=0,86 ; \mathrm{Syx}_{\mathrm{y}}=0,375$. 
Les droites de régression représentant les relations entre l'incorporation de $\mathrm{P}$ et les métabolismes azoté et glucidique sont représentées sur les figures 1, 2, 3 et 4 . Nous avons calculé, pour chacune d'entre elles, les intervalles de confiance au niveau $95 \%$ de la moyenne des valeurs prédites par les équations (zones pointillées).

La quantité de phosphore incorporé par les bactéries du rumen est positivement corrélée avec :

- la disparition de l'azote ammoniacal du milieu, "équation $A$ " :

$\mathrm{Pi}(\mathrm{mg})=0,162 \mathrm{~N}-\mathrm{NH}_{3}(\mathrm{mg})+0,376$

$r=0,90$ (fig. 1) ;

- I'accroissement des protéines bactériennes, "équation B " :

$\mathrm{Pi}(\mathrm{mg})=0,166 \mathrm{~N}$-prot. $(\mathrm{mg})+0,355$

$r=0,86$ (fig. 2) ;

- la production d'acides gras volatils, " équation $C$ " :

$\mathrm{Pi}(\mathrm{mg})=0,586 \mathrm{AGV}$ (mmole) - 0,004

$r=0,89$ (fig. 3$)$;

- la matière organique fermentée "équation $D$ »:

$\mathrm{Pi}(\mathrm{mg})=0,00672$ (MOF) (mg) $+0,01181$

$r=0,89$ (fig. 4$)$.

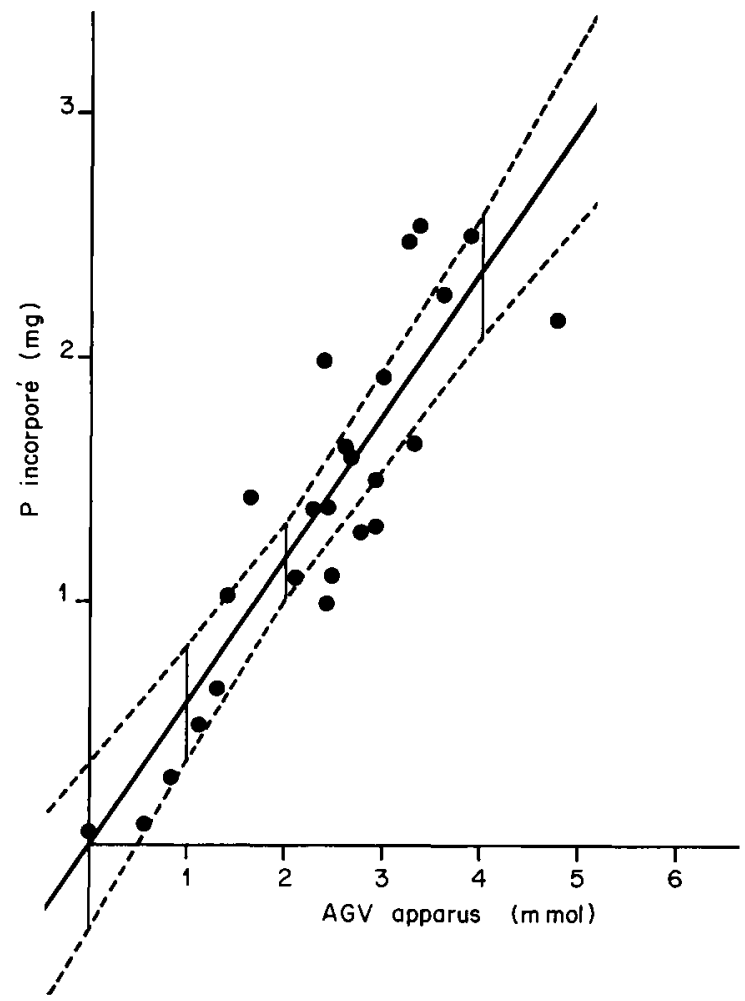

FIG. 3. - Relation entre l'incorporation de phosphore dans les bactéries (calculée à partir du ${ }^{32} \mathrm{P}$ ) et la production d'acides gras volatils.

$\mathrm{Pi}_{\text {(mg) }}=0,586 \mathrm{AGV}_{\text {(mmoles) }}-0,004$.

$\mathrm{n}=25 ; \mathrm{r}=0,89 ;$ Syx $=0,331$. 


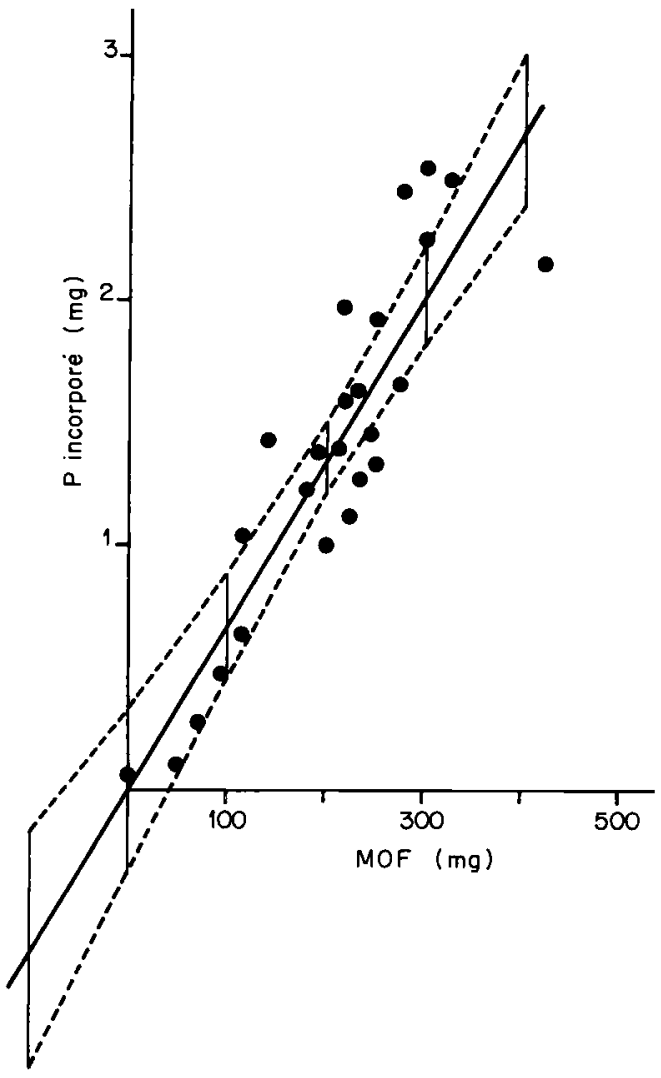

FIG. 4. - Relation entre l'incorporation de phosphore dans les bactéries (calculée à partir du ${ }^{32} \mathrm{P}$ ) et la quantité de matière organique fermentée.

$\mathrm{Pi}_{(\mathrm{mg})}=0,00672 \mathrm{MOF}_{(\mathrm{mg})}+0,01181$.

$\mathrm{n}=25 ; \mathrm{r}=0,89 ;$ Syx $=0,334$.

\section{Discussion.}

Bien que nous ayons incubé des faibles volumes et que nous ayons utilisé des contenus de rumen filtrés comme inoculum, nous avons obtenu un rendement de synthèse protéique moyen s'élevant à $30,2 \pm 6,2 \mathrm{~g}$ de $\mathrm{N}-\mathrm{NH}_{3}$ utilisé et $31,1 \pm 1,9 \mathrm{~g}$ de $\mathrm{N}$-protéique synthétisé par $\mathrm{kg}$ de MOF. Ces deux valeurs sont sensiblement identiques aux rendements moyens cités dans la littérature (Jarrige et al., 1978 ; ARC, 1980).

1. Relations entre l'incorporation de phosphore, la protéosynthèse et la production d'acides gras volatils.

II existe une corrélation élevée entre l'incorporation de phosphore et l'incorporation d'azote, corrélation qui est légèrement supérieure pour $\mathrm{N}-\mathrm{NH}_{3}$ utilisé que pour $\mathrm{N}$-protéique synthétisé.

Reproduction Nutrition Développement, $n^{\circ}$ 4-1983. -7 . 
On note que, dans le cas d'absence de substrat énergétique où la lyse bactérienne peut être égale ou supérieure à la croissance (bilan apparent de $\mathrm{N}$ protéique nul ou négatif), l'incorporation de phosphore se poursuit. Les droites de régression reliant le phosphore incorporé par les bactéries (Pi) et $\mathrm{N}-\mathrm{NH}_{3}$ utilisé ou $\mathrm{N}$-protéique synthétisé ne passent pas par l'origine. On peut observer, pour une utilisation de $\mathrm{N}-\mathrm{NH}_{3}$ et une synthèse protéique nulles, une incorporation moyenne de $0,37 \mathrm{mg}$ de phosphore. Ce phénomène peut s'expliquer de deux manières :

- un processus de fermentation sans croissance bactérienne notable se produirait dans certains essais (carence en soufre ou en substrats glucidiques), les conditions pour un découplage de fermentation étant réunies; des bactéries pourraient aussi se trouver en phase de latence et produire encore des AGV sans protéosynthèse. Dans ce cas, le phosphore incorporé servirait pour les besoins enzymatiques de la glycolyse ou serait mis en réserve dans les cellules sous forme de phosphate inorganique et ne serait pas relié à la protéosynthèse ;

- d'après Van Nevel et Demeyer (1977), certaines bactéries pourraient se lyser, dans nos milieux d'incubation, libérant ainsi des composés azotés comme le $\mathrm{N}-\mathrm{NH}_{3} ;$ d'autres bactéries, dans le même temps, continueraient à proliférer en incorporant de l'azote et du phosphore et en produisant des AGV. La plupart de nos essais étant réalisés en $3 \mathrm{~h}$, les bactéries qui incorporent le phosphore radioactif ne se lysent probablement pas dans un temps aussi court. Dans de telles conditions, l'incorporation de phosphore mesurée par ${ }^{32} \mathrm{P}$ correspondrait à une croissance totale. Les quantités mesurées de $\mathrm{N}-\mathrm{NH}_{3}$ utilisé ou de $\mathrm{N}$-protéique synthétisé correspondraient à la différence entre croissance " totale » et lyse et refléteraient seulement une croissance " nette ».

Pour estimer l'incorporation nette de phosphore, on pourrait admettre que l'ordonnée à l'origine s'élimine en tenant compte d'une libération de phosphore due à la lyse. On aurait alors comme équations :

$$
\begin{aligned}
& \text { A }^{\prime} \mathrm{Pi}(\mathrm{mg})=0,162 \mathrm{~N}-\mathrm{NH}_{3}(\mathrm{mg}), \\
& \text { B' }^{\prime} \mathrm{Pi}(\mathrm{mg})=0,166 \mathrm{~N} \text {-prot. }(\mathrm{mg}) .
\end{aligned}
$$

Les deux valeurs du rapport $N$ incorporé/ $P$ incorporé calculées d'après les équations $A$ et $A^{\prime}$ (5,0 pour l'incorporation totale de $P$ et 6,2 pour l'incorporation nette), sont nettement inférieures aux valeurs $N / P$ des culots bactériens pris chez des animaux à jeun $(8,13 \pm 0,26$ dans nos conditions avec régime purifié et $8,37 \pm 0,75$ d'après Van Nevel et Demeyer, 1977). Par contre, elles se rapprochent de celles calculées d'après les résultats de Czerkawski $(1976)(5,3)$ sur des contenus de rumen prélevés chez des animaux à différents moments de la journée, ce qui prouve que, malgré la lyse bactérienne, les équations que nous avons définies pourraient correspondre à des conditions de croissance bactérienne assez proches des conditions in vivo.

Les équations $C$ et $D$ qui relient le phosphore incorporé aux AGV produits dans le milieu d'incubation ou à $M O F$, montrent que la droite de régression passe par l'origine : en l'absence de fermentation glucidique, il n'y a pas d'incorporation de phosphore, contrairement à ce que nous avons observé pour l'utilisation du N-NH . 
2. Estimation des besoins en phosphore, in vitro et in vivo.

L'utilisation des équations obtenues pour estimer les besoins en phosphore n'est réellement valable que pour des milieux où les conditions, de lyse ou de nombre de bactéries en phase de latence, sont identiques à celles de nos milieux d'incubation, c'est-à-dire pour un rapport "croissance nette »/ " croissance totale " identique. Cependant, en l'absence de données précises dans la littérature, nous utiliserons tout de même ces équations pour obtenir des valeurs approchées des besoins en $P$, soit d'après les relations entre $P$ incorporé et protéosynthèse ou entre $P$ incorporé et fermentations glucidiques.

In vitro, dans les conditions d'incubation adoptées par Kumaresan (1976) ou Beaumatin (1981), les quantités de $\mathrm{N}-\mathrm{NH}_{3}$ utilisé varient entre 20 et $40 \mathrm{mg} \mathrm{N} / 100 \mathrm{ml}$ de milieu selon que les donneurs d'inoculum sont des moutons adultes ou des agneaux. Les besoins " totaux " en phosphore seraient alors de 4 à 7,2 $\mathrm{mg}$ et les besoins "nets " de 3,2 à $6,4 \mathrm{mg}$ par $100 \mathrm{ml}$ de milieu pour une durée de $3 \mathrm{~h}$ d'incubation. Dans ces mêmes milieux, la quantité d'AGV produits s'élève généralement de 8,5 à $10 \mathrm{mmoles}$ selon l'âge des animaux donneurs. On note, d'après l'équation $C$, une incorporation de $0,59 \mathrm{mg} \mathrm{P} / \mathrm{mmole} \mathrm{d}^{\prime} A G V$ produite. Les incorporations se situeraient entre 5 et $5,9 \mathrm{mg} / 100 \mathrm{ml}$ de milieu pour $3 \mathrm{~h}$ d'incubation, valeurs du même ordre que celles obtenues avec l'équation $A^{\prime}$. Ces valeurs, comprises entre 30 et $70 \mathrm{mg} /$ litre, correspondent aux estimations citées précédemment pour la croissance de culture pure de bactéries ou pour l'activité cellulolytique.

Cependant, nos estimations concernent du phosphore réellement incorporé, et les besoins pourraient être supérieurs à ces valeurs. En effet, avec des inocula provenant d'animaux carencés en phosphore qui apportaient $65 \mathrm{mg}$ de $\mathrm{P} / \mathrm{I}$ de milieu, Bonilla (1976) a montré que l'activité fermentaire était accrue par addition de phosphore $(300 \mathrm{mg} / \mathrm{l})$; de même, nous avons stimulé l'utilisation de $\mathrm{N}-\mathrm{NH}_{3}$ $(+15 \%)$ et la production d'AGV $(+5 \%)$ par une supplémentation en $P(100 \mathrm{mg} / \mathrm{l})$ d'un milieu én contenant $70 \mathrm{mg} / \mathrm{l}$ (résultats non publiés). Or, avec des régimes subcarencés en phosphore, des valeurs encore plus faibles peuvent être observées in vivo (Durand et al., 1982).

In vivo, si les conditions étaient identiques aux conditions in vitro, en particulier en ce qui concerne la lyse, on pourrait, d'après les valeurs cie $\mathrm{N}$-protéique synthétisé, estimer les besoins en phosphore des bactéries du rumen. La quantité d'azote microbien synthétisé dans le rumen se situe aux environs de $30 \mathrm{~g} / \mathrm{kg}$ de matière organique apparemment digérée dans le rumen (MOaDR) (Jarrige et al., 1978 ; ARC, 1980); en adoptant cette valeur moyenne de $30 \mathrm{~g}$, les besoins " nets " en phosphore pour les bactéries du rumen, calculés comme nous avons vu précédemment, pourraient être de $4,9 \mathrm{~g}$ et les besoins " totaux " de 6,0 g. Environ $65 \%$ de la matière organique digestible (MOD) sont apparemment digérés dans le rumen (Jarrige et al., 1978 et $A R C, 1980$ ) ce qui porte les besoins en phosphore, estimés par rapport à la MOD, à $3,2 \mathrm{~g} / \mathrm{kg}$ (besoins « nets ») et à $3,9 \mathrm{~g} / \mathrm{kg}$ (besoins " totaux »). Cependant, des rendements de synthèse protéique nettement plus élevés (Armstrong, 1980) sont parfois observés. Dans le cas d'un rendement de synthèse très élevé $(40 \mathrm{~g} \mathrm{~N} / \mathrm{kg}$ MOaDR par exemple), les besoins 
" nets » en phosphore seraient de 4,2 g et les besoins « totaux " de $5,2 \mathrm{~g} / \mathrm{kg}$ MOD.

En tenant compte de l'équation $\mathrm{D}$ reliant l'incorporation de $\mathrm{P}$ à la matière organique fermentée, les besoins seraient de $6,7 \mathrm{~g}$ de $\mathrm{P} / \mathrm{kg} \mathrm{MOF}$, soit $4,4 \mathrm{~g}$ de $\mathrm{P} / \mathrm{kg}$ MOD. Cette estimation est un peu plus élevée qu'avec l'équation reliant le phosphore à la protéosynthèse, lorsque son rendement est moyen. Cependant, lorsque celui-ci est important, les deux modes d'estimation donnent des résultats voisins.

\section{Conclusion.}

En utilisant le phosphore radioactif, nous avons pu évaluer l'incorporation de $P$ dans les microorganismes et la relier aux métabolismes azoté et glucidique. Dans des incubations de $3 \mathrm{~h}$, les incorporations maximales s'élèvent à $70 \mathrm{mg}$ $\mathrm{P} /$ litre de milieu. Cependant, les apports en phosphore disponible devraient être légèrement supérieurs et seront précisés dans des études ultérieures. Les besoins in vivo que nous avons déduits sont assez approximatifs et peuvent varier selon l'intensité de la fermentation, le rendement de la protéosynthèse ou l'importance relative des bactéries en phase de latence ou en état de lyse, cette dernière pouvant libérer du phosphore dans le milieu. Ces besoins se situeraient entre 3,0 et $5,0 \mathrm{~g} / \mathrm{kg}$ de matière organique digestible et les teneurs en phosphore disponible devraient être supérieures à $70 \mathrm{mg} / \mathrm{l}$. Ces valeurs feront l'objet de vérifications in vivo.

Reçu en novembre 1982. Accepté en février 1983.

\section{Références}

AGRICULTURAL RESEARCH COUNCIL, 1980. The Nutrient requirements of ruminant livestock, 121-181. Ed. Commonwealth Agricultural Bureau, London.

ARMSTRONG D. G., 1980. Net efficiencies in vivo of microbial $\mathbf{N}$ synthesis in ruminant livestock, 400-413. In H. J. OSLAGE, K. BOUR. Protein metabolism and nutrition. Proc. 3rd E.A.A.P. Symp. Prot. Metab. Nutr. Braunschweig, May 1980.

BEAUMATIN Ph., 1981. Influence du phosphore alimentaire sur la digestion et la protéosynthèse microbiennes dans le rumen du mouton. Thèse Univ. Toulouse, $110 \mathrm{pp}$.

BONILLA S. E., 1976. Phosphorus in the nutrition of sheep: composition of body fluids, microbial fermentation and feed intake. Thesis, California Davis University, $176 \mathrm{pp}$.

CZERKAWSKI J. W., 1976. Chemical composition of microbial matter in the rumen. J. Sci. Food Agric., 27, 621-632.

DEMEYER D. I., 1981. Rumen microbes and digestion of plant cell walls. Agric. Environ., 6. 295-337.

DURAND M., KAWASHIMA R., 1980. Influence of minerals in rumen microbial digestion, 375-408. In RUCKEBUSCH Y., THIVEND P., Proc. 5th int. Symp. Ruminant Physiol., ClermontFerrand, 3rd-7th sept. 1979. MTP Press Ltd. Lancaster.

DURAND M., BERTIER B., HANNEQUART G., GUÉGUEN L., 1982. Influence d'une subcarence en phosphore et d'un excès de calcium alimentaire sur la phosphatémie et les teneurs en phosphore et calcium des contenus de rumen du mouton. Reprod. Nutr. Dévelop., 22, 865-879.

HUNGATE R. E., 1966. The rumen and its microbes. Acad. Press Inc., New York. 
JARRIGE R., JOURNET M., VERITE R., CHAMPREDON C., PION R., 1978. Azote, 89-128. In Alimentation des ruminants, éd. INRA Publications, Versailles.

KUMARESAN A., 1976. Interactions entre le zinc et les microorganismes du rumen chez le mouton recevant de l'urée comme source unique d'azote. Thèse Univ. Toulouse, $96 \mathrm{pp}$.

LEVY, J., CAMPBELL J. J., BLACKBURN T. H., 1973. Introductory microbiology, p. 59. J. Wiley \& Sons, New York.

MARTINEZ A., 1972. Effect of some major and trace element interactions upon in vitro rumen cellulose digestion. Thesis Oregon State Univ., $71 \mathrm{pp}$.

OTTENSTEIN D. M., BARTLEY D. A., 1971. Improved gas chromatography separation of free acids $\mathrm{C}_{2}-\mathrm{C}_{5}$ in dilution solution. Anal. Chem., 43, 952-955.

PALFII F., DEM'YANCHUK G. T., YATSKANICH M. M., TYCHKA B. D., 1976. Diurnal changes in concentration of phosphorus compounds in rumen bacteria of young bulls given concentrate mixtures three times and twice daily. Dokl. Vsesoy. Ord. Lenina Akad Sel'skokh Nauk., (10), 32-34.

SAIZEVA G. N., BELOZERSKY A. N., NOVOJILOVA L. P., 1959. Phosphorus compounds of Azotobacter vinelandii in the course of development of the culture. Biokhimia, 24, 1054-1065.

STEWART C. S., 1975. Some effects of phosphate and volatile fatty acid salts on the growth of rumen bacteria. J. gen. Microbiol., 89, 319-326.

TEMPEST D. W., 1969. Quantitative relationships between inorganic cations and anionic polymers in growing bacteria, 87-111. In P. M. MEADOW, S. J. PIRT, Microbial growth, 9th Symp. Soc. gen. Microbiol. London, April 1969. Vol. 19. Cambr. Univ. Press, New York.

VAN NEVEL C. J., DEMEYER D. I., 1977. Determination of rumen microbial growth in vitro from ${ }^{32} \mathrm{P}$ labelled phosphate incorporation. Br. J. Nutr., 38, 101-114. 